\title{
Excitation of Localized Plasmons in Metal Nanoshell and Nanotube with Dielectric Cores
}

\author{
Masakazu Ichikawa ${ }^{\dagger}$ \\ Department of Applied Physics and Quantum-Phase Electronics Center, Graduate School of Engineering, The University of Tokyo, 7-3-1 Hongo, \\ Bunkyo-ku, Tokyo 113-8656, Japan \\ †Corresponding author: ichikawa@ap.t.u-tokyo.ac.jp
}

Received: 28 May, 2021; Accepted: 12 September, 2021; Published: 18 September, 2021

Metal nanoshells and nanotubes with dielectric cores are useful structures to change the surface plasmon frequencies in the wide range. Here, our localized plasmon theory derived in the random phase approximation at high frequency condition is applied to investigate the localized plasmon excitations for metal nanoshells and nanotubes with dielectric cores, which are embedded in dielectrics. Assuming that local dielectric functions for metals and dielectrics have step function shapes at the metal and dielectric interfaces in the quasi-static approximation, analytical formulas can be derived for the localized plasmon excitation. It is found that the bonding and antibonding localized surface plasmons are excited when the nanoshell and nanotube have finite thicknesses and that the surface plasmon frequencies can be controlled in the wide range by changing the ratio of the inner radius to the outer one of the

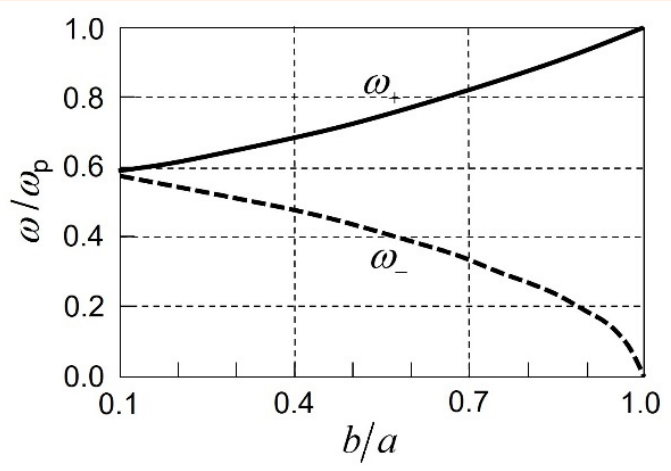
nanoshell and nanotube. The localized surface plasmons, however, are not excited in the zero-thickness limit, i.e., two-dimensional shell where only the lights are emitted from the interfaces of dielectric cores and surrounding dielectrics.

Keywords Surface plasmon; Bulk plasmon; Light emission; Nanoshell; Nanotube; Dielectrics

\section{INTRODUCTION}

The localized surface plasmons (LSPs) can couple with photons to much enhance their local electric fields near metal nanostructures at the surface plasmon excitation energies [1-5]. These effects caused by LSPs can be used to fabricate nanoscale photonic devices and to perform very high-sensitive optical measurements. It is desired for these applications to tune the surface plasmon energies in the wide range by modifying metal nanostructures.

Metal nanospheres with a dielectric core-shell structure, i.e., nanoshells with dielectric cores, have attracted attention due to its excellent and tunable sensing properties for optical measurements [6-13]. Metal nanowires with a dielectric core-shell structure, i.e., nanotubes with dielectric cores and carbon nanotubes, have also attracted attention due to the same reason [14-19].

Up to now, many theoretical studies on the localized surface plasmon excitation for metal nanoshells [9-13] and nanotubes [14-18] have been reported, where it is shown that the plasmon hybridization of the inner and outer surfaces and the shell thickness change of the metal nanostructures can tune the surface plasmon energies in the wide range.

The author has developed several theories on the localized plasmons in two- and three-dimensional metal nanostructures using the random phase approximation (RPA) at high frequency conditions in vacuum [20-24] and also developed theories on the localized plasmons for metal nanostructures in dielectrics $[25,26]$. They have been recently applied to investigate the localized plasmon excitations for metal nanostructures in dielectrics by focused electron beams [27].

In this study, theoretical studies are reported on the localized plasmon excitations for metal nanoshells and nanotubes with dielectric cores, which are embedded in dielectrics. Analytical formulas are derived for the localized plasmon excitation and light emission from the nanoshell and nanotube structures. 


\section{EQUATIONS FOR LOCALIZED PLASMONS IN METAL NANOSTRUCTURES}

\section{A. Scalar potential in the quasi-static approximation}

In our previous studies [20, 25], the following integral equation for the effective scalar potential $\varphi_{\text {eff }}(\boldsymbol{r}, \omega)$ and the external scalar potential $\varphi_{\text {ext }}(\boldsymbol{r}, \omega)$ for the metal nanostructures in dielectrics has been derived in the RPA at the high frequency condition, i.e., the plasmon excitation condition, using the quasi-static approximation where the retardation effect on the scalar potential and the vector potential are ignored:

$$
\left[1-\frac{\omega_{\mathrm{p}}^{2}(\boldsymbol{r})}{\omega(\omega+i \gamma)}+4 \pi \chi_{\mathrm{d}}(\boldsymbol{r}, \omega)\right] \varphi_{\mathrm{eff}}(\boldsymbol{r}, \omega)+\int \mathrm{d} \boldsymbol{r}_{1} \varphi_{\mathrm{eff}}\left(\boldsymbol{r}_{1}, \omega\right) \nabla_{1} \frac{1}{\left|\boldsymbol{r}-\boldsymbol{r}_{1}\right|} \cdot \nabla_{1}\left[\frac{\omega_{\mathrm{p}}^{2}\left(\boldsymbol{r}_{1}\right)}{4 \pi \omega(\omega+i \gamma)}-\chi_{\mathrm{d}}\left(\boldsymbol{r}_{1}, \omega\right)\right]=\varphi_{\mathrm{ext}}(\boldsymbol{r}, \omega),
$$

where $\omega_{\mathrm{p}}\left(\boldsymbol{r}_{1}\right)=\sqrt{4 \pi e^{2} n\left(\boldsymbol{r}_{1}\right) / m_{\mathrm{e}}}$ is the local bulk plasmon frequency, $m_{\mathrm{e}}$ and $-e$ are the electron mass and charge, respectively, and $\chi_{\mathrm{d}}\left(\boldsymbol{r}_{1}, \omega\right)$ is the electric susceptibility of the dielectrics with the local position dependence and uniformity or cubic symmetry, which can be expressed by the position-dependent scalar. $\gamma$ is the plasmon decay frequency caused by the finite lifetime of the single electron in the metal nanostructures $[2,28]$. The Gaussian units are used in this article. $n\left(\boldsymbol{r}_{1}\right)$ is the local electron density in metal nanostructures, which is given using quantum mechanical calculations by

$$
n\left(\boldsymbol{r}_{1}\right)=2 \sum_{n}\left|\Psi_{n}\left(\boldsymbol{r}_{1}\right)\right|^{2} \theta\left(E_{\mathrm{F}}-E_{n}\right)
$$

where $\Psi_{n}\left(\boldsymbol{r}_{1}\right)$ is the normalized single-electron wave function with an energy $E_{n}$ in metal nanostructures, $E_{\mathrm{F}}$ is the Fermi energy, and $\theta\left(E_{\mathrm{F}}-E_{n}\right)$ is the step function.

The quasi-static approximation is valid when the metal nanostructure size is much smaller than the wavelength of light and its amplitude can be regarded as constant in the nanostructure, i.e., in the case of $k a \ll 1$ where $k$ is the wave number of light and is the typical metal nanostructure size. For example, $k a$ becomes about 0.1 when the wavelength of light and the nanostructure size are assumed to be $500 \mathrm{~nm}$ (a typical wavelength used in the plasmonics) and $10 \mathrm{~nm}$, respectively. Thus, Eq. (1) is considered an effective formula to study the localized plasmon excitations in the metal nanostructures with less than about $10 \mathrm{~nm}$ in size.

As shown in our previous study [20], the non-local dielectric function $\varepsilon\left(\boldsymbol{r}, \boldsymbol{r}_{1}, \omega\right)$ is defined using Eq. (1) by

$$
\begin{aligned}
& \int \varepsilon\left(\boldsymbol{r}, \boldsymbol{r}_{1}, \omega\right) \varphi_{\mathrm{eff}}\left(\boldsymbol{r}_{1}, \omega\right) \mathrm{d} \boldsymbol{r}_{1}=\varphi_{\mathrm{ext}}(\boldsymbol{r}, \omega), \\
& \varepsilon\left(\boldsymbol{r}, \boldsymbol{r}_{1}, \omega\right)=\left[1-\frac{\omega_{\mathrm{p}}^{2}\left(\boldsymbol{r}_{1}\right)}{\omega(\omega+i \gamma)}+4 \pi \chi_{\mathrm{d}}\left(\boldsymbol{r}_{1}, \omega\right)\right] \delta\left(\boldsymbol{r}-\boldsymbol{r}_{1}\right)+\nabla_{1} \frac{1}{\left|\boldsymbol{r}-\boldsymbol{r}_{1}\right|} \cdot \nabla_{1}\left[\frac{\omega_{\mathrm{p}}^{2}\left(\boldsymbol{r}_{1}\right)}{4 \pi \omega(\omega+i \gamma)}-\chi_{\mathrm{d}}\left(\boldsymbol{r}_{1}, \omega\right)\right] .
\end{aligned}
$$

The first and second terms of the dielectric function are related to the localized bulk and surface plasmons, respectively, making it possible to investigate the correlation between these plasmons.

By taking the Laplacian $\nabla^{2}$ of both sides of Eq. (1), the following equation and local dielectric function $\varepsilon(\boldsymbol{r}, \omega)$ are derived for the electric displacement $\boldsymbol{D}(\boldsymbol{r}, \omega)=-\nabla \varphi_{\text {ext }}(\boldsymbol{r}, \omega)$ and the electric field $\boldsymbol{E}(\boldsymbol{r}, \omega)=-\nabla \varphi_{\text {eff }}(\boldsymbol{r}, \omega)$ without external charges:

$$
\begin{aligned}
& \nabla \cdot \boldsymbol{D}(\boldsymbol{r}, \omega)=\nabla \cdot[\varepsilon(\boldsymbol{r}, \omega) \boldsymbol{E}(\boldsymbol{r}, \omega)]=0, \\
& \varepsilon(\boldsymbol{r}, \omega)=1-\frac{\omega_{\mathrm{p}}^{2}(\boldsymbol{r})}{\omega(\omega+i \gamma)}+4 \pi \chi_{\mathrm{d}}(\boldsymbol{r}, \omega) .
\end{aligned}
$$

The equation of $\nabla \cdot[\varepsilon(\boldsymbol{r}, \omega) \boldsymbol{E}(\boldsymbol{r}, \omega)]=0$ becomes the Laplace equation for the effective scalar potential $\varphi_{\text {eff }}(\boldsymbol{r}, \omega)$ when the local dielectric function has no position dependence.

The same local dielectric function in Eq. (4) can be also derived when the retardation effect and the vector potential in the electric field are considered $[24,25]$. This indicates that our formula is non-local for the scalar potential but local for the electric field. After the derivation of Eq. (1) for metal nanostructures in a vacuum [20], Eq. (1) has been numerically calculated to investigate the quantum effect on the localized surface plasmons in metal nanospheres using the local electron density $n\left(\boldsymbol{r}_{1}\right)$ derived by quantum mechanical method [29]. This indicates that Eq. (1) can investigate the quantum effect on the localized plasmons. Our recent study has also shown that the quantum effect can be investigated using the local dielectric function in Eq. (4) [30].

Equation (1) has several advantages that the correlation between the localized bulk and surface plasmons and the quantum effect on the localized plasmons can be studied. Furthermore, the results can be derived by considering only the scalar potential without considering the vector potential, and analytical results with the dipole and multipole terms can be derived in several cases as shown below. 


\section{B. Dipole moment and light emission intensity}

The electric dipole moment is given in the quasi-static approximation by $[21,25]$

$$
\begin{aligned}
& \boldsymbol{p}(\omega)=-\int \mathrm{d} \boldsymbol{r}_{1} \varphi_{\mathrm{eff}}\left(\boldsymbol{r}_{1}, \omega\right) \nabla_{1}\left[\frac{\omega_{\mathrm{p}}^{2}\left(\boldsymbol{r}_{1}\right)}{4 \pi \omega(\omega+i \gamma)}-\chi_{\mathrm{d}}\left(\boldsymbol{r}_{1}, \omega\right)\right], \\
& \ddot{\boldsymbol{p}}(\omega)=-\omega^{2} \boldsymbol{p}(\omega) .
\end{aligned}
$$

where $\ddot{\boldsymbol{p}}(\omega)$ is the second order derivative with respect to time of the dipole moment. When metal nanostructures exist in dielectrics without optical absorption, the emitted photon number or light emission intensity with energy $\hbar \omega$ per unit solid angle $\Omega$ in the $\boldsymbol{r}$ direction from nanostructures is given in the electric dipole approximation by [21, 25]

$$
\begin{aligned}
I_{\mathrm{ph}}(\Omega, \omega) & =\frac{1}{4 \pi^{2} c_{\mathrm{d}}^{3} \hbar \omega}\left|\boldsymbol{e}_{r} \times \ddot{\boldsymbol{p}}(\omega)\right|^{2} \\
& =\frac{\omega^{3}}{4 \pi^{2} c_{\mathrm{d}}^{3} \hbar}\left|\boldsymbol{e}_{r} \times \int \mathrm{d} \boldsymbol{r}_{1} \varphi_{\mathrm{eff}}\left(\boldsymbol{r}_{1}, \omega\right) \nabla_{1}\left[\frac{\omega_{\mathrm{p}}^{2}\left(\boldsymbol{r}_{1}\right)}{4 \pi \omega(\omega+i \gamma)}-\chi_{\mathrm{d}}\left(\boldsymbol{r}_{1}, \omega\right)\right]\right|^{2}
\end{aligned}
$$

where $c_{\mathrm{d}}$ is the light velocity in the dielectrics and $\boldsymbol{e}_{r}=\boldsymbol{r} / \boldsymbol{r}$ is the unit vector in the $\boldsymbol{r}$ direction. Since the electric multipole contribution to the light emission intensity is proportional to $(k a)^{2(l-1)}(l \geq 2)[21,27]$, which is much smaller than that of the dipole one $(l=1)$ in the case $k a \ll 1$, Eq. (6) is an effective formula to calculate the total light emission intensity from the metal nanostructures with less than about $10 \mathrm{~nm}$ in size.

\section{APPLICATION TO METAL NANOSTRUCTURE WITH DIELECTRIC CORE}

\section{A. Metal nanoshell with dielectric core}

\section{Scalar potential}

In the quasi-static approximation, the localized plasmon excitation is studied for a metal nanoshell with the outer radius $a$ and the inner radius $b$ having a dielectric core, as shown in Figure 1. The local electron density of the nanoshell and susceptibility of the dielectrics are assumed to have the following step function shapes:

$$
\begin{aligned}
& n\left(\boldsymbol{r}_{1}\right)=n_{0}\left[\theta\left(a-r_{1}\right)-\theta\left(b-r_{1}\right)\right], \\
& \chi_{\mathrm{d}}\left(\boldsymbol{r}_{1}, \omega\right)=\chi_{\mathrm{s}}(\omega) \theta\left(r_{1}-a\right)+\chi_{\mathrm{c}}(\omega) \theta\left(b-r_{1}\right),
\end{aligned}
$$

where $n_{0}, \chi_{\mathrm{s}}(\omega), \chi_{\mathrm{c}}(\omega)$, and $\theta(x)$ are the constant electron density, the uniform electric susceptibilities of surrounding and core dielectrics, and the step function, respectively. The scalar potential and the Coulomb potential in Eq. (1) are expanded using the spherical harmonics $Y_{l m}(\theta, \phi)$ as

$$
\begin{aligned}
& \varphi_{\begin{array}{c}
\mathrm{eff} \\
\mathrm{ext}
\end{array}}(\boldsymbol{r}, \omega)=\sum_{l=0}^{\infty} \sum_{m=-l}^{l} \varphi_{\begin{array}{c}
\mathrm{eff} \\
\mathrm{ext}
\end{array}}^{l m}(r, \omega) Y_{l m}(\theta, \phi), \\
& \frac{1}{\left|\boldsymbol{r}-\boldsymbol{r}_{1}\right|}=\sum_{l=0}^{\infty} \sum_{m=-l}^{l} \frac{4 \pi}{2 l+1} Y_{l m}(\theta, \phi) Y_{l m}^{*}\left(\theta_{1}, \phi_{1}\right) \frac{r_{<}^{l}}{r_{>}^{l+1}},
\end{aligned}
$$

where $r_{<}=\min \left(r, r_{1}\right)$ and $r_{>}=\max \left(r, r_{1}\right)$.

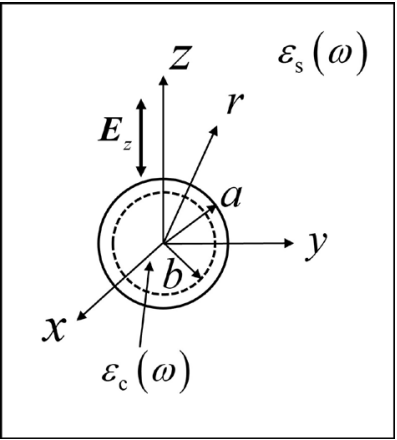

Figure 1: Geometrical arrangement of the metal nanoshell with dielectric core in the dielectric. 
The previous study [31] has reported using quantum mechanical calculations that the local electron density in metal nanoshell with about $10 \mathrm{~nm}$ in size has a structure similar to the step function shape. The Friedel oscillation of the electron density also appears in the nanoshell due to the quantum interference effect. However, the period of the oscillation is much smaller than the wavelength of the light and the amplitude of the oscillation is small, which results in a small contribution to the plasmon excitation [32]. Thus, the results can be obtained in good approximation by using the local electron density with the step function shape, which makes it also possible to obtain analytical results for the localized plasmons. However, this assumption may fail for the metal nanoshell with less than $1 \mathrm{~nm}$ in size where the oscillation with a large amplitude appears due to the quantum interference effect $[33,34]$. Equation (1) should be numerically solved using the local electron density $n\left(\boldsymbol{r}_{1}\right)$ to examine the validity of the step function-like electron density for tiny metal nanostructures.

Our study considers not only the electric dipole $(l=1)$ case but also the multipole one $(l \geq 2)$ as shown below. In the dipole case, our study using step function-like dielectric functions is equivalent to the previous studies [12, 13]. In these studies, the Laplace equations have been solved in the quasi-static and dipole cases using the boundary conditions for the electric displacement and the electric field, the Drude dielectric function for the nanoshell, and uniform dielectric functions for the dielectrics. This is because Eq. (1) is equivalent to the Laplace equation when the local dielectric function is uniform as discussed in Section II.A.

Substituting Eqs. (7) and (8) into Eq. (1), the following equations are derived using the relation $d \theta\left(a-r_{1}\right) / d r_{1}=-\delta\left(r_{1}-a\right)$, the constant bulk plasmon frequency $\omega_{\mathrm{p}}=\sqrt{4 \pi e^{2} n_{0} / m_{\mathrm{e}}}$, and so forth, whose detailed calculations are shown in Appendix A:

$$
\begin{aligned}
& \varphi_{\mathrm{eff}}^{\operatorname{lm}}(a, \omega)=\frac{1}{|A|}\left\{\left[1-\frac{\omega_{\mathrm{p}}^{2}(l+1)}{\omega(\omega+i \gamma)(2 l+1)}+\frac{4 \pi \chi_{\mathrm{c}}(\omega) l}{2 l+1}\right] \varphi_{\mathrm{ext}}^{\operatorname{lm}}(a, \omega)\right. \\
& \left.-\frac{l}{2 l+1}\left(\frac{b}{a}\right)^{l+1}\left[\frac{\omega_{\mathrm{p}}^{2}}{\omega(\omega+i \gamma)}+4 \pi \chi_{\mathrm{c}}(\omega)\right] \varphi_{\mathrm{ext}}^{l m}(b, \omega)\right\}, \\
& \varphi_{\mathrm{eff}}^{\operatorname{lm}}(b, \omega)=\frac{1}{|A|}\left\{\left[1-\frac{\omega_{\mathrm{p}}^{2} l}{\omega(\omega+i \gamma)(2 l+1)}+\frac{4 \pi \chi_{\mathrm{s}}(\omega)(l+1)}{2 l+1}\right] \varphi_{\mathrm{ext}}^{\operatorname{lm}}(b, \omega)\right. \\
& \left.-\frac{l+1}{2 l+1}\left(\frac{b}{a}\right)^{l}\left[\frac{\omega_{\mathrm{p}}^{2}}{\omega(\omega+i \gamma)}+4 \pi \chi_{\mathrm{s}}(\omega)\right] \varphi_{\mathrm{ext}}^{l m}(a, \omega)\right\},
\end{aligned}
$$

where $|A|$ is the determinant that is given by

$$
|A|=\left|\begin{array}{cc}
1-\frac{\omega_{\mathrm{p}}^{2} l}{\omega(\omega+i \gamma)(2 l+1)}+\frac{4 \pi \chi_{\mathrm{s}}(\omega)(l+1)}{2 l+1} & \frac{l}{2 l+1}\left(\frac{b}{a}\right)^{l+1}\left[\frac{\omega_{\mathrm{p}}^{2}}{\omega(\omega+i \gamma)}+4 \pi \chi_{\mathrm{c}}(\omega)\right] \\
\frac{l+1}{2 l+1}\left(\frac{b}{a}\right)^{l}\left[\frac{\omega_{\mathrm{p}}^{2}}{\omega(\omega+i \gamma)}+4 \pi \chi_{\mathrm{s}}(\omega)\right] & 1-\frac{\omega_{\mathrm{p}}^{2}(l+1)}{\omega(\omega+i \gamma)(2 l+1)}+\frac{4 \pi \chi_{\mathrm{c}}(\omega) l}{2 l+1}
\end{array}\right| .
$$

The localized surface plasmon frequencies are derived by $|A|=0$, which gives the quadratic equation for $\omega(\omega+i \gamma)$. When the nanoshell with dielectric core exists in a vacuum or air, i.e., $\chi_{\mathrm{s}}(\omega)=0$, the surface plasmon frequencies are given ignoring the plasmon decay $\gamma=0$ by

$$
\begin{gathered}
\omega_{ \pm}=\omega_{\mathrm{p}} \sqrt{\frac{1+\frac{4 \pi \chi_{\mathrm{c}}(\omega) l}{(2 l+1)^{2}}\left[l+(l+1)\left(\frac{b}{a}\right)^{2 l+1}\right] \pm D}{2\left[1+\frac{4 \pi \chi_{\mathrm{c}}(\omega) l}{2 l+1}\right]}} \\
\because D=\sqrt{\left\{1+\frac{4 \pi \chi_{\mathrm{c}}(\omega) l}{(2 l+1)^{2}}\left[l+(l+1)\left(\frac{b}{a}\right)^{2 l+1}\right]\right\}^{2}-4\left[1+\frac{4 \pi \chi_{\mathrm{c}}(\omega) l}{2 l+1}\right]\left[1-\left(\frac{b}{a}\right)^{2 l+1}\right] \frac{l(l+1)}{(2 l+1)^{2}}} .
\end{gathered}
$$

When the nanoshell has no dielectric core, i.e., $\chi_{\mathrm{c}}(\omega)=0$, the surface plasmon frequencies are given by 


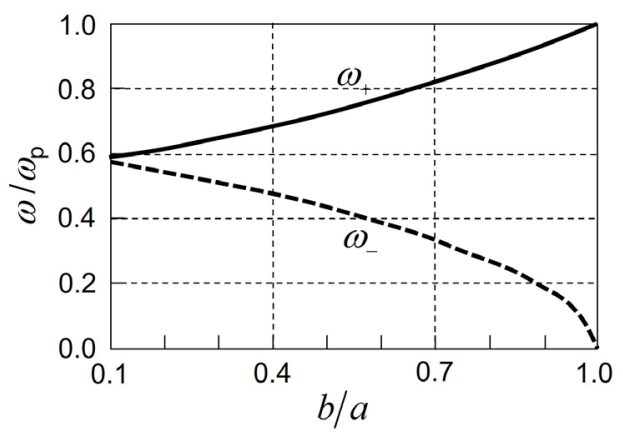

Figure 2: $\omega / \omega_{\mathrm{p}}$ as a function of $b / a$ at $l=1$ for a nanoshell with a silica core in a vacuum. Solid and dashed lines show the antibonding $\omega_{+}$and bonding $\omega_{-}$surface plasmon modes, respectively.

$$
\omega_{ \pm}=\frac{\omega_{\mathrm{p}}}{\sqrt{2}} \sqrt{1 \pm \frac{1}{2 l+1} \sqrt{1+4 l(l+1)\left(\frac{b}{a}\right)^{2 l+1}}},
$$

which coincide with the previous ones derived by the semi-classical and quantum mechanical methods [9, 10].

Figure 2 shows $\omega / \omega_{\mathrm{p}}$ as a function of $b / a$ at an electric dipole case $l=1$ in Eq. (12) for a silica $\left(\mathrm{SiO}_{2}\right)$ dielectric core; $\varepsilon_{\mathrm{c}}(\omega)=1+4 \pi \chi_{\mathrm{c}}(\omega)=3.9$, where $\varepsilon_{\mathrm{c}}(\omega)$ is the uniform dielectric function, because only the dipole surface plasmons at $l=1$ contribute to the light emission as shown below. The $b / a$ dependence of the frequency coincides qualitatively with the previous results [9-11]. There are two surface plasmon dipole modes; a symmetrically coupled (bonding) mode $\omega_{-}$and an anti-symmetrically coupled (antibonding) mode $\omega_{+}$, that are caused by the surface plasmon hybridization at inner and outer surfaces in the metal nanoshell as shown in the previous studies [9-11]. The hybridization of the inner and outer surface plasmons increases as the metal nanoshell thickness becomes smaller, i.e., $b / a$ becomes larger. When $b / a=1$, meaning the zero-thickness limit of the nanoshell, the frequencies of the bonding mode $\omega_{-}$and antibonding mode $\omega_{+}$become zero and $\omega_{\mathrm{p}}$, respectively. These results indicate that the surface plasmon frequencies can be controlled in the wide range by changing the $b / a$ ratio.

\section{Dipole moment and light emission intensity}

The electric dipole moment is given using Eq. (5) by

$$
\begin{aligned}
\ddot{\boldsymbol{p}}(\omega)=-\boldsymbol{e}_{z} \omega^{2}\left\{2 \pi a^{2}\left[\frac{\omega_{\mathrm{p}}^{2}}{4 \pi \omega(\omega+i \gamma)}+\chi_{\mathrm{s}}(\omega)\right] \sum_{l=0}^{\infty} \sqrt{\frac{2 l+1}{4 \pi}} \varphi_{\mathrm{eff}}^{l 0}(a, \omega)\right. \\
\left.\quad-2 \pi b^{2}\left[\frac{\omega_{\mathrm{p}}^{2}}{4 \pi \omega(\omega+i \gamma)}+\chi_{\mathrm{c}}(\omega)\right] \sum_{l=0}^{\infty} \sqrt{\frac{2 l+1}{4 \pi}} \varphi_{\mathrm{eff}}^{l 0}(b, \omega)\right\} \int_{-1}^{1} \mathrm{~d}(\cos \theta) P_{l}^{0}(\cos \theta) \cos \theta \\
=-\boldsymbol{e}_{z} \sqrt{\frac{4 \pi}{3}} \omega^{2}\left\{a^{2}\left[\frac{\omega_{\mathrm{p}}^{2}}{4 \pi \omega(\omega+i \gamma)}+\chi_{\mathrm{s}}(\omega)\right] \varphi_{\mathrm{eff}}^{10}(a, \omega)-b^{2}\left[\frac{\omega_{\mathrm{p}}^{2}}{4 \pi \omega(\omega+i \gamma)}+\chi_{\mathrm{c}}(\omega)\right] \varphi_{\mathrm{eff}}^{10}(b, \omega)\right\},
\end{aligned}
$$

where $\boldsymbol{e}_{z}$ is the unit vector in the $z$ direction, the rotational symmetry of the system around the $z$ axis $(m=0)$ and the orthogonal property of the associated Legendre function $P_{1}^{0}(\cos \theta)=\cos \theta$ are used. This indicates that only the terms of $l=1($ electric dipole) at $r=a$ and $r=b$ contributes to the dipole moment.

The external scalar potentials are given by $\varphi_{\text {ext }}^{10}(a, \omega)=-\sqrt{4 \pi / 3} E_{z} a$ and $\varphi_{\text {ext }}^{10}(b, \omega)=-\sqrt{4 \pi / 3} E_{z} b$, which are derived by $\varphi_{\text {ext }}(\boldsymbol{r}, \omega)=-E_{z} z=-E_{z} r \cos \theta$ in the quasi-static approximation. The emitted photon number is calculated using Eqs. (6) and (14) by

$$
I_{\mathrm{ph}}(\Omega, \omega)=\frac{\omega^{3} a^{4} \sin ^{2} \theta}{48 \pi^{3} c_{\mathrm{d}}^{3} \hbar}\left|\frac{\omega_{\mathrm{p}}^{2}}{\omega(\omega+i \gamma)}\left[\varphi_{\mathrm{eff}}^{10}(a, \omega)-\left(\frac{b}{a}\right)^{2} \varphi_{\mathrm{eff}}^{10}(b, \omega)\right]+4 \pi\left[\chi_{\mathrm{s}}(\omega) \varphi_{\mathrm{eff}}^{10}(a, \omega)-\left(\frac{b}{a}\right)^{2} \chi_{\mathrm{c}}(\omega) \varphi_{\mathrm{eff}}^{10}(b, \omega)\right]\right|^{2},
$$

where $\theta$ is the angle between the unit vectors $\boldsymbol{e}_{r}$ and $\boldsymbol{e}_{z}$. 
The contribution of the two surface plasmon modes having $\omega_{ \pm}$to the light emission becomes smaller as the nanoshell becomes thinner. This is because $\varphi_{\text {eff }}^{10}(b, \omega)$ gradually approaches to $\varphi_{\text {eff }}^{1 \frac{10}{0}}(a, \omega)$ and the first term related to the plasmon excitation in Eq. (15) becomes smaller when $b / a \rightarrow 1$ at fixed $a$. This term finally disappears using the relation $\varphi_{\text {eff }}^{10}(a, \omega)=\varphi_{\text {eff }}^{10}(b, \omega)$ at $b / a=1$. However, the second term related to the dielectrics in Eq. (15) still remains for $\chi_{\mathrm{s}}(\omega) \neq \chi_{\mathrm{c}}(\omega)$ at $b / a=1$. The effective scalar potential at $b / a=1$, i.e., the zero-thickness limit of the nanoshell is given using Eqs. (9) and (10) by

$$
\varphi_{\mathrm{eff}}^{\operatorname{lm}}(a, \omega)=\varphi_{\mathrm{eff}}^{\operatorname{lm}}(b, \omega)=\frac{\varphi_{\mathrm{ext}}^{\operatorname{lm}}(a, \omega)}{1+4 \pi \chi_{\mathrm{s}}(\omega) \frac{l+1}{2 l+1}+4 \pi \chi_{\mathrm{c}}(\omega) \frac{l}{2 l+1}}
$$

where the term of $1-\omega_{\mathrm{p}}^{2} /[\omega(\omega+i \gamma)]$ that gives the antibonding surface plasmon frequency $\omega_{\mathrm{p}}$ appears in the numerator and denominator of Eq. (16) but is cancelled with each other. Equation (16) indicates that the bonding $\omega_{-}$and antibonding $\omega_{+}$surface plasmons do not contribute to the light emission. Only the surrounding dielectric $\chi_{\mathrm{s}}(\omega)$ with cavity structure and the core dielectric $\chi_{\mathrm{c}}(\omega)$ with sphere structure contribute the light emission at the zero-thickness limit of the nanoshell.

\section{B. Metal nanotube with dielectric core}

\section{Scalar potential}

In the quasi-static approximation, the localized plasmon excitation is studied for a metal nanotube with the outer radius $a$ and the inner radius $b$ having a dielectric core as shown Figure 3. The local electron density of the nanotube and susceptibility of the dielectrics are assumed to have the following step function shapes:

$$
\begin{aligned}
& n\left(\boldsymbol{r}_{1}\right)=n_{0}\left[\theta\left(a-\rho_{1}\right)-\theta\left(b-\rho_{1}\right)\right], \\
& \chi_{\mathrm{d}}\left(\boldsymbol{r}_{1}, \omega\right)=\chi_{\mathrm{s}}(\omega) \theta\left(\rho_{1}-a\right)+\chi_{\mathrm{c}}(\omega) \theta\left(b-\rho_{1}\right) .
\end{aligned}
$$

The scalar potential and the Coulomb potential in Eq. (1) are expanded using the cylindrical coordinate as

$$
\begin{aligned}
& \varphi_{\mathrm{ext}}^{\mathrm{eff}}(\boldsymbol{\rho}, z, \omega)=\frac{1}{2 \pi} \sum_{m=-\infty}^{\infty} e^{i m \phi} \int \mathrm{d} k \varphi_{\mathrm{eff}}^{m}(\rho, k, \omega) e^{i k z}, \\
& \frac{1}{\left|\boldsymbol{r}-\boldsymbol{r}_{1}\right|}=\frac{1}{\pi} \sum_{m=-\infty}^{\infty} e^{i m\left(\phi-\phi_{1}\right)} \int_{-\infty}^{\infty} \mathrm{d} q I_{m}\left(q \rho_{<}\right) K_{m}\left(q \rho_{>}\right) e^{i q\left(z-z_{1}\right)},
\end{aligned}
$$

where $\boldsymbol{r}=(\boldsymbol{\rho}, z), m$ is integer, $\phi$ is the azimuth angle in the $\boldsymbol{\rho}=(x, y)$ plane, $\rho_{<}=\min \left(\rho, \rho_{1}\right), \rho_{>}=\max \left(\rho, \rho_{1}\right)$, and $I_{m}(s)$ and $K_{m}(s)$ are the modified Bessel functions of the first and second kinds, respectively [16, 25$]$.

Substituting Eqs. (17) and (18) into Eq. (1), the following equations are derived using the relation like $d \theta\left(a-\rho_{1}\right) / d r_{1}=$ $-\delta\left(\rho_{1}-a\right)$, whose detailed calculations are shown in Appendix B:

$$
\begin{array}{r}
\varphi_{\mathrm{eff}}^{m}(a, k, \omega)=\frac{1}{|B|}\left\{\left[1+\frac{\omega_{\mathrm{p}}^{2}}{\omega(\omega+i \gamma)} k b I_{m}(k b) K_{m}^{\prime}(k b)+4 \pi \chi_{\mathrm{c}}(\omega) k b I_{m}^{\prime}(k b) K_{m}(k b)\right] \varphi_{\mathrm{ext}}^{m}(a, k, \omega)\right. \\
\left.-k b I_{m}^{\prime}(k b) K_{m}(k a)\left[\frac{\omega_{\mathrm{p}}^{2}}{\omega(\omega+i \gamma)}+4 \pi \chi_{\mathrm{c}}(\omega)\right] \varphi_{\mathrm{ext}}^{m}(b, k, \omega)\right\}
\end{array}
$$

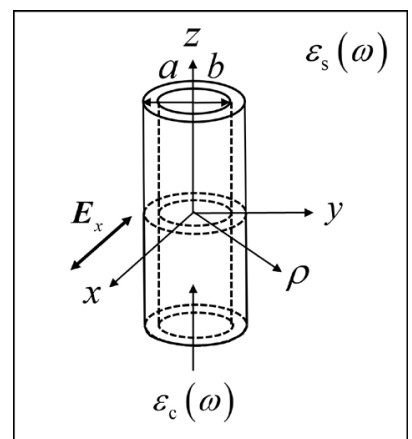

Figure 3: Geometrical arrangement of the metal nanotube with dielectric core in the dielectric. 


$$
\begin{aligned}
\varphi_{\mathrm{eff}}^{m}(b, k, \omega)=\frac{1}{|B|}\{ & k a I_{m}(k b) K_{m}^{\prime}(k a)\left[\frac{\omega_{\mathrm{p}}^{2}}{\omega(\omega+i \gamma)}+4 \pi \chi_{\mathrm{s}}(\omega)\right] \varphi_{\mathrm{ext}}^{m}(a, k, \omega) \\
& \left.+\left[1-\frac{\omega_{\mathrm{p}}^{2}}{\omega(\omega+i \gamma)} k a I_{m}^{\prime}(k a) K_{m}(k a)-4 \pi \chi_{\mathrm{s}}(\omega) k a I_{m}(k a) K_{m}^{\prime}(k a)\right] \varphi_{\mathrm{ext}}^{m}(b, k, \omega)\right\},
\end{aligned}
$$

where $|B|$ is a determinant that is given by

$$
|B|=\left|\begin{array}{rr}
1-\frac{\omega_{\mathrm{p}}^{2}}{\omega(\omega+i \gamma)} k a I_{m}^{\prime}(k a) K_{m}(k a) & k b I_{m}^{\prime}(k b) K_{m}(k a)\left[\frac{\omega_{\mathrm{p}}^{2}}{\omega(\omega+i \gamma)}+4 \pi \chi_{\mathrm{c}}(\omega)\right] \\
-4 \pi \chi_{\mathrm{s}}(\omega) k a I_{m}(k a) K_{m}^{\prime}(k a) & \\
-k a I_{m}(k b) K_{m}^{\prime}(k a)\left[\frac{\omega_{\mathrm{p}}^{2}}{\omega(\omega+i \gamma)}+4 \pi \chi_{\mathrm{s}}(\omega)\right] & 1+\frac{\omega_{\mathrm{p}}^{2}}{\omega(\omega+i \gamma)} k b I_{m}(k b) K_{m}^{\prime}(k b) \\
+4 \pi \chi_{\mathrm{c}}(\omega) k b I_{m}^{\prime}(k b) K_{m}(k b)
\end{array}\right|,
$$

where $I_{m}^{\prime}(s)$ and $K_{m}^{\prime}(s)$ are the first-order derivatives of the modified Bessel functions.

The localized surface plasmon frequencies are derived by $|B|=0$, which gives the quadratic equation for $\omega(\omega+i \gamma)$. When the nanotube with dielectric core exists in vacuum or air, i.e., $\chi_{\mathrm{s}}(\omega)=0$, the surface plasmon frequencies are given ignoring the plasmon decay $\gamma=0$ at $m=1$ and $k=0$ by

$$
\omega_{ \pm}=\omega_{\mathrm{p}} \sqrt{\frac{1+\pi \chi_{\mathrm{c}}(\omega)\left[1+\left(\frac{b}{a}\right)^{2}\right] \pm \sqrt{\left\{1+\pi \chi_{\mathrm{c}}(\omega)\left[1+\left(\frac{b}{a}\right)^{2}\right]\right\}^{2}-\left[1+2 \pi \chi_{\mathrm{c}}(\omega)\right]\left[1-\left(\frac{b}{a}\right)^{2}\right]}}{2+4 \pi \chi_{\mathrm{c}}(\omega)}}
$$

where the relations of $\lim _{s \rightarrow 0}\left[s I_{1}^{\prime}(s) K_{1}(s)\right]=1 / 2, \lim _{s \rightarrow 0}\left[s I_{1}(s) K_{1}^{\prime}(s)\right]=-1 / 2$, or others are used. When the nanotube has no dielectric core, i.e., $\chi_{\mathrm{c}}(\omega)=0$, the surface plasmon frequencies are given by

$$
\omega_{ \pm}=\omega_{\mathrm{p}} \sqrt{\frac{1 \pm b / a}{2}} .
$$

Figure 4 shows $\omega / \omega_{\mathrm{p}}$ as a function of $b / a$ in Eq. (22) for a $\mathrm{SiO}_{2}$ dielectric core; $\varepsilon_{\mathrm{c}}(\omega)=1+4 \pi \chi_{\mathrm{c}}(\omega)=3.9$, where $\varepsilon_{\mathrm{c}}(\omega)$ is an uniform silica dielectric function, because only the surface plasmons at $m=1$ and $k=0$ contribute to the light emission as shown below. The $b / a$ dependence of the frequency coincides qualitatively with the previous results $[14,15]$. There are also two surface plasmon dipole modes; a symmetrically coupled (bonding) mode $\omega_{-}$and an anti-symmetrically coupled (antibonding) mode $\omega_{+}$that are caused by the surface plasmon hybridization at inner and outer surfaces in the metal nanotube as shown in the previous studies $[14,15]$. The hybridization of the inner and outer surface plasmons increases when the metal nanotube thickness becomes smaller, i.e., $b / a$ becomes larger. When $b / a=1$, meaning the zero-thickness limit of the nanotube, the frequencies of the bonding mode $\omega_{-}$and antibonding mode $\omega_{+}$become zero and $\omega_{\mathrm{p}}$, respectively. These results indicate that the surface plasmon frequencies can be controlled in the wide range by changing the $b / a$ ratio.

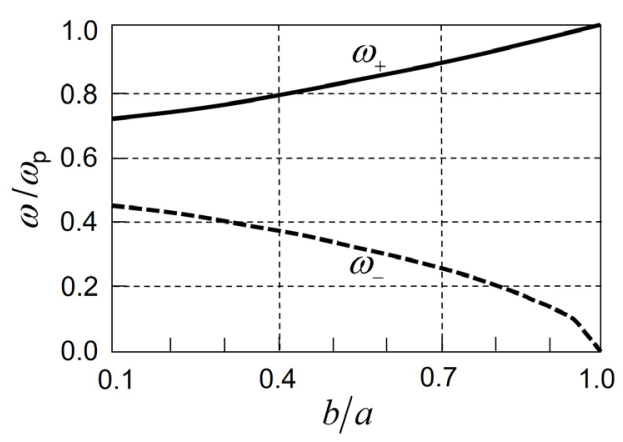

Figure 4: $\omega / \omega_{\mathrm{p}}$ as a function of $b / a$ at $k=0$ and $m=1$ for nanotube with a silica core in a vacuum. Solid and dashed lines show the antibonding $\omega_{+}$and bonding $\omega_{-}$surface plasmon modes, respectively. 


\section{Dipole moment and light emission intensity}

The electric dipole moment is given using Eq. (5) by

$$
\begin{aligned}
\ddot{\boldsymbol{p}}(\omega) & =-\boldsymbol{e}_{x} \frac{\omega^{2}}{4 \pi} \lim _{k \rightarrow 0}\left\{a\left[\frac{\omega_{\mathrm{p}}^{2}}{\omega(\omega+i \gamma)}+4 \pi \chi_{\mathrm{s}}(\omega)\right] \sum_{m=-\infty}^{\infty} \varphi_{\mathrm{eff}}^{m}(a, k, \omega)\right. \\
& \left.-b\left[\frac{\omega_{\mathrm{p}}^{2}}{\omega(\omega+i \gamma)}+4 \pi \chi_{\mathrm{c}}(\omega)\right] \sum_{m=-\infty}^{\infty} \varphi_{\mathrm{eff}}^{m}(b, k, \omega)\right\} \int_{0}^{2 \pi} e^{i m \phi} \cos \phi \mathrm{d} \phi \\
& =\boldsymbol{e}_{x} \frac{\omega^{2}}{2}\left\{\frac{\omega_{\mathrm{p}}^{2}}{\omega(\omega+i \gamma)}\left[-a \varphi_{\mathrm{eff}}^{1}(a, 0, \omega)+b \varphi_{\mathrm{eff}}^{1}(b, 0, \omega)\right]-4 \pi\left[a \chi_{\mathrm{s}}(\omega) \varphi_{\mathrm{eff}}^{1}(a, 0, \omega)-b \chi_{\mathrm{c}}(\omega) \varphi_{\mathrm{eff}}^{1}(b, 0, \omega)\right]\right\},
\end{aligned}
$$

where $\boldsymbol{e}_{x}$ is the unit vector in the $x$ direction. The term at $m=1$ (electric dipole) and $k=0$ only contributes to the dipole moment, which is derived by using the translational invariance in the $z$ direction and the mirror symmetry in the $y$ direction of the system.

The external scalar potentials are given by $\varphi_{\text {ext }}^{1}(a, 0, \omega)=-E_{x} a L$ and $\varphi_{\text {ext }}^{1}(b, 0, \omega)=-E_{x} b L$, which are derived by $\varphi_{\text {ext }}(\rho, z, \omega)=-E_{x} x=-E_{x} \rho \cos \phi$ in the quasi-static approximation and $L$ is the length of the nanotube. The emitted photon number is calculated using Eqs. (6) and (24) by

$$
\begin{aligned}
& I_{\mathrm{ph}}(\Omega, \omega)=\frac{\omega^{3} a^{2} \sin ^{2} \theta}{16 \pi^{2} c_{\mathrm{d}}^{3} \hbar} \mid \frac{\omega_{\mathrm{p}}^{2}}{\omega(\omega+i \gamma)} {\left[\varphi_{\mathrm{eff}}^{1}(a, 0, w)-\frac{b}{a} \varphi_{\mathrm{eff}}^{1}(b, 0, w)\right] } \\
&+\left.4 \pi\left[\chi_{s}(\omega) \varphi_{\mathrm{eff}}^{1}(a, 0, \omega)-\frac{b}{a} \chi_{c}(\omega) \varphi_{\mathrm{eff}}^{1}(b, 0, \omega)\right]\right|^{2},
\end{aligned}
$$

where $\theta$ is the angle between the unit vectors $\boldsymbol{e}_{r}$ and $\boldsymbol{e}_{x}$.

The contribution of the two surface plasmon modes having $\omega_{ \pm}$to the light emission becomes smaller as the nanotube become thinner. This is because $\varphi_{\text {eff }}^{1}(b, 0, \omega)$ approaches to $\varphi_{\text {eff }}^{1}(a, 0, \omega)$ and the first term related to the plasmon excitation in Eq. (25) becomes smaller when $b / a \rightarrow 1$ at fixed $a$. This term finally disappears using the relation $\varphi_{\text {eff }}^{1}(a, 0, \omega)=\varphi_{\text {eff }}^{1}(b, 0, \omega)$ at $b / a=1$. However, the second term related to the dielectrics in Eq. (25) still remains for $\chi_{\mathrm{s}}(\omega) \neq \chi_{\mathrm{c}}(\omega)$ at $b / a=1$.

The effective scalar potential at $b / a=1$, i.e., the zero-thickness limit of the nanotube is given using Eqs. (19) and (20) by

$$
\varphi_{\mathrm{eff}}^{m}(a, k, \omega)=\varphi_{\mathrm{eff}}^{m}(b, k, \omega)=\frac{\varphi_{\mathrm{ext}}^{m}(a, k, \omega)}{1-4 \pi \chi_{\mathrm{s}}(\omega) k a I_{m}(k a) K_{m}^{\prime}(k a)+4 \pi \chi_{\mathrm{c}}(\omega) k a I_{m}^{\prime}(k a) K_{m}(k a)},
$$

where the term of $1-\omega_{\mathrm{p}}^{2} /[\omega(\omega+i \gamma)]$ that gives the antibonding surface plasmon frequency $\omega_{\mathrm{p}}$ appears in the numerator and denominator of Eq. (26) but is cancelled with each other. Equation (26) indicates that the bonding $\omega_{-}$and antibonding $\omega_{+}$surface plasmons do not contribute to the light emission at the zero-thickness limit. Only the surrounding dielectric $\chi_{\mathrm{s}}(\omega)$ with cave structure and the core dielectric $\chi_{\mathrm{c}}(\omega)$ with wire structure contribute the light emission at $b / a=1$.

\section{CONCLUSIONS}

The localized plasmon theory derived in the random phase approximation at a high frequency condition was applied to investigate the localized plasmon excitations for metal nanoshells and nanotubes with dielectric cores, which were embedded in dielectrics. Assuming that local dielectric functions for metals and dielectrics had step function shapes at the metal and dielectric interfaces in the quasi-static approximation, analytical formulas were derived for the localized plasmon excitation and light emission intensities. The bonding and antibonding localized surface plasmons were excited when the nanoshell and nanotube had finite thicknesses and the surface plasmon frequencies could be controlled in the wide range by changing the ratio of the inner radius to the outer one of the nanoshell and nanotube. The localized surface plasmons, however, were not excited in the zero-thickness limit where only the lights were emitted from the interfaces of the dielectric cores and the surrounding dielectrics.

The formulas reported here can be easily developed to study the localized plasmons in multi-shell metal nanostructures. This indicates that our theory is useful to study the localized plasmons in complex metal nanostructures.

\section{Acknowledgments}

This study was supported by Department of Applied Physics, The University of Tokyo. 


\section{Appendix A}

Substituting Eqs. (7) and (8) into Eq. (1), the following equations are derived using $\varepsilon_{\mathrm{c}}(\omega)=1+4 \pi \chi_{\mathrm{c}}(\omega)$ and $\varepsilon_{\mathrm{s}}(\omega)=1+4 \pi \chi_{\mathrm{s}}(\omega)$, where $\varepsilon_{\mathrm{c}}(\omega)$ and $\varepsilon_{\mathrm{s}}(\omega)$ are uniform dielectric functions for core and surrounding dielectrics, respectively:

(1) Outside the nanoshell: $r>a$,

$$
\begin{aligned}
\varepsilon_{\mathrm{s}}(\omega) \varphi_{\mathrm{eff}}^{l m}(r, \omega)- & \frac{l}{2 l+1}\left(\frac{a}{r}\right)^{l+1}\left[\frac{\omega_{\mathrm{p}}^{2}}{\omega(\omega+i \gamma)}+4 \pi \chi_{\mathrm{s}}(\omega)\right] \varphi_{\mathrm{eff}}^{l m}(a, \omega) \\
& +\frac{l}{2 l+1}\left(\frac{b}{r}\right)^{l+1}\left[\frac{\omega_{\mathrm{p}}^{2}}{\omega(\omega+i \gamma)}+4 \pi \chi_{\mathrm{c}}(\omega)\right] \varphi_{\mathrm{eff}}^{l m}(b, \omega)=\varphi_{\mathrm{ext}}^{l m}(r, \omega) .
\end{aligned}
$$

(2) Inside the nanoshell: $b \leq r \leq a$,

$$
\begin{aligned}
{\left[1-\frac{\omega_{\mathrm{p}}^{2}}{\omega(\omega+i \gamma)}\right] \varphi_{\mathrm{eff}}^{l m}(r, \omega)+} & \frac{l+1}{2 l+1}\left(\frac{r}{a}\right)^{l}\left[\frac{\omega_{\mathrm{p}}^{2}}{\omega(\omega+i \gamma)}+4 \pi \chi_{\mathrm{s}}(\omega)\right] \varphi_{\mathrm{eff}}^{l m}(a, \omega) \\
& +\frac{l}{2 l+1}\left(\frac{b}{r}\right)^{l+1}\left[\frac{\omega_{\mathrm{p}}^{2}}{\omega(\omega+i \gamma)}+4 \pi \chi_{\mathrm{c}}(\omega)\right] \varphi_{\mathrm{eff}}^{l m}(b, \omega)=\varphi_{\mathrm{ext}}^{l m}(r, \omega)
\end{aligned}
$$

(3) Inside the dielectric core: $0 \leq r<b$,

$$
\begin{aligned}
\varepsilon_{\mathrm{c}}(\omega) \varphi_{\mathrm{eff}}^{\operatorname{lm}}(r, \omega)+ & \frac{l+1}{2 l+1}\left(\frac{r}{a}\right)^{l}\left[\frac{\omega_{\mathrm{p}}^{2}}{\omega(\omega+i \gamma)}+4 \pi \chi_{\mathrm{s}}(\omega)\right] \varphi_{\mathrm{eff}}^{l m}(a, \omega) \\
& -\frac{l+1}{2 l+1}\left(\frac{r}{b}\right)^{l}\left[\frac{\omega_{\mathrm{p}}^{2}}{\omega(\omega+i \gamma)}+4 \pi \chi_{\mathrm{c}}(\omega)\right] \varphi_{\mathrm{eff}}^{\operatorname{lm}}(b, \omega)=\varphi_{\mathrm{ext}}^{\operatorname{lm}}(r, \omega) .
\end{aligned}
$$

Equation (A1) coincides with Eq. (A2) at $r=a$, indicating that the bulk plasmon contribution that is the first term in Eq. (A2) disappears at the interface between the surrounding dielectrics and the metal nanoshell by the surface plasmon screening effect on the bulk plasmon, which is caused by the correlation between the bulk and surface plasmons. Then, a linear equation for the effective scalar potentials of $\varphi_{\text {eff }}^{l m}(a, \omega)$ and $\varphi_{\mathrm{eff}}^{\operatorname{lm}}(b, \omega)$ is derived at $r=a$.

Equation (A2) also coincides with Eq. (A3) at $r=b$, indicating that the bulk plasmon contribution disappears at the interface between the core dielectrics and the metal nanoshell by the surface plasmon screening effect. Another linear equation for the effective scalar potentials of $\varphi_{\mathrm{eff}}^{l m}(a, \omega)$ and $\varphi_{\mathrm{eff}}^{l m}(b, \omega)$ is derived at $r=b$. Then, Eqs. (9) and (10) are derived using the Cramer's rule to solve the two simultaneous linear equations.

The determinant $|A|$ in Eq. (11) is given by

$$
\begin{aligned}
|A|=\alpha & +\frac{\omega_{\mathrm{p}}^{2} \beta}{\omega(\omega+i \gamma)}+\frac{\omega_{\mathrm{p}}^{4} \eta}{[\omega(\omega+i \gamma)]^{2}}, \\
\because \quad \eta= & \frac{l(l+1)}{(2 l+1)^{2}}\left[1-\left(\frac{b}{a}\right)^{2 l+1}\right], \\
& \beta=-\left\{1+4 \pi \chi_{\mathrm{s}}(\omega)\left(\frac{l+1}{2 l+1}\right)^{2}+4 \pi \chi_{\mathrm{c}}(\omega)\left(\frac{l}{2 l+1}\right)^{2}+\frac{l(l+1)}{(2 l+1)^{2}}\left(\frac{b}{a}\right)^{2 l+1}\left[4 \pi \chi_{\mathrm{s}}(\omega)+4 \pi \chi_{\mathrm{c}}(\omega)\right]\right\}, \\
& \alpha=1+4 \pi \chi_{\mathrm{s}}(\omega)\left(\frac{l+1}{2 l+1}\right)+4 \pi \chi_{\mathrm{c}}(\omega) \frac{l}{2 l+1}+(4 \pi)^{2} \chi_{\mathrm{s}}(\omega) \chi_{\mathrm{c}}(\omega) \frac{l(l+1)}{(2 l+1)^{2}}\left[1-\left(\frac{b}{a}\right)^{2 l+1}\right] .
\end{aligned}
$$

The determinant $|A|=0$ gives the quadratic equation for $\omega(\omega+i \gamma)$ for localized surface plasmon frequencies that are given by

$$
\omega_{ \pm}\left(\omega_{ \pm}+i \gamma\right)=\omega_{\mathrm{p}}^{2} \frac{-\beta \pm \sqrt{\beta^{2}-4 \alpha \eta}}{2 \alpha}
$$

When the nanoshell with the dielectric core exists in vacuum or air, i.e., $\chi_{\mathrm{s}}(\omega)=0$, the surface plasmon frequencies are given ignoring the plasmon decay $\gamma=0$ by Eq. (12).

\section{Appendix B}

Substituting Eqs. (17) and (18) into Eq. (1), the following equations are derived using $\varepsilon_{\mathrm{c}}(\omega)$ and $\varepsilon_{\mathrm{s}}(\omega)$; uniform dielectric functions for core and surrounding dielectrics, respectively: 
(1) Outside the nanotube: $\rho>a$,

$$
\begin{aligned}
\varepsilon_{\mathrm{s}}(\omega) \varphi_{\mathrm{eff}}^{m}(\rho, k, \omega)- & {\left[\frac{\omega_{\mathrm{p}}^{2}}{\omega(\omega+i \gamma)}+4 \pi \chi_{\mathrm{s}}(\omega)\right] k a I_{m}^{\prime}(k a) K_{m}(k \rho) \varphi_{\mathrm{eff}}^{m}(a, k, \omega) } \\
& +\left[\frac{\omega_{\mathrm{p}}^{2}}{\omega(\omega+i \gamma)}+4 \pi \chi_{\mathrm{c}}(\omega)\right] k b I_{m}^{\prime}(k b) K_{m}(k \rho) \varphi_{\mathrm{eff}}^{m}(b, k, \omega)=\varphi_{\mathrm{ext}}^{m}(\rho, k, \omega) .
\end{aligned}
$$

(2) Inside the nanotube: $b \leq \rho \leq a$,

$$
\begin{gathered}
{\left[1-\frac{\omega_{\mathrm{p}}^{2}}{\omega(\omega+i \gamma)}\right] \varphi_{\mathrm{eff}}^{m}(\rho, k, \omega)-\left[\frac{\omega_{\mathrm{p}}^{2}}{\omega(\omega+i \gamma)}+4 \pi \chi_{\mathrm{s}}(\omega)\right] k a I_{m}(k \rho) K_{m}^{\prime}(k a) \varphi_{\mathrm{eff}}^{m}(a, k, \omega)} \\
+\left[\frac{\omega_{\mathrm{p}}^{2}}{\omega(\omega+i \gamma)}+4 \pi \chi_{\mathrm{c}}(\omega)\right] k b I_{m}^{\prime}(k b) K_{m}(k \rho) \varphi_{\mathrm{eff}}^{m}(b, k, \omega)=\varphi_{\mathrm{ext}}^{m}(\rho, k, \omega)
\end{gathered}
$$

(3) Inside the dielectric core: $0 \leq \rho<b$,

$$
\begin{aligned}
\varepsilon_{\mathrm{c}}(\omega) \varphi_{\mathrm{eff}}^{m}(\rho, k, \omega)- & {\left[\frac{\omega_{\mathrm{p}}^{2}}{\omega(\omega+i \gamma)}+4 \pi \chi_{\mathrm{s}}(\omega)\right] k a I_{m}(k \rho) K_{m}^{\prime}(k a) \varphi_{\mathrm{eff}}^{m}(a, k, \omega) } \\
& +\left[\frac{\omega_{\mathrm{p}}^{2}}{\omega(\omega+i \gamma)}+4 \pi \chi_{\mathrm{c}}(\omega)\right] k b I_{m}(k \rho) K_{m}^{\prime}(k b) \varphi_{\mathrm{eff}}^{m}(b, k, \omega)=\varphi_{\mathrm{ext}}^{m}(\rho, k, \omega) .
\end{aligned}
$$

Using the relation of $I_{m}(s) K_{m}^{\prime}(s)-I_{m}^{\prime}(s) K_{m}(s)=-1 / s$, Eq. (B1) coincides with Eq. (B2) at $\rho=a$, indicating that the bulk plasmon contribution that is the first term in Eq. (B2) disappears at the interface between the surrounding dielectrics and the metal nanotube by the surface plasmon screening effect. Then, a linear equation for the effective scalar potentials of $\varphi_{\text {eff }}^{m}(a, k, \omega)$ and $\varphi_{\text {eff }}^{m}(b, k, \omega)$ is derived at $\rho=a$.

Equation (B2) also coincides with Eq. (B3) at $\rho=b$, indicating that the bulk plasmon contribution disappears at the interface between the core dielectrics and the metal nanotube by the surface plasmon screening effect. Another linear equation for the effective scalar potentials of $\varphi_{\mathrm{eff}}^{m}(a, k, \omega)$ and $\varphi_{\mathrm{eff}}^{m}(b, k, \omega)$ is derived at $\rho=b$. Then, Eqs. (19) and (20) are derived using the Cramer's rule to solve two simultaneous linear equations.

The determinant $|B|$ in Eq. (21) is given by

$$
\begin{aligned}
|B|=\alpha & +\frac{\omega_{\mathrm{p}}^{2} \beta}{\omega(\omega+i \gamma)}+\frac{\omega_{\mathrm{p}}^{4} \eta}{[\omega(\omega+i \gamma)]^{2}} \\
\because \quad \eta= & k a k b\left[I_{m}^{\prime}(k b) K_{m}(k a) I_{m}(k b) K_{m}^{\prime}(k a)-I_{m}^{\prime}(k a) K_{m}(k a) I_{m}(k b) K_{m}^{\prime}(k b)\right], \\
\beta= & k b I_{m}(k b) K_{m}^{\prime}(k b)-k a I_{m}^{\prime}(k a) K_{m}(k a)-4 \pi \chi_{\mathrm{c}}(\omega) k a k b I_{m}^{\prime}(k a) K_{m}(k a) I_{m}^{\prime}(k b) K_{m}(k b) \\
& -4 \pi \chi_{\mathrm{s}}(\omega) k a k b I_{m}(k a) K_{m}^{\prime}(k a) I_{m}(k b) K_{m}^{\prime}(k b)+\left[4 \pi \chi_{\mathrm{s}}(\omega)+4 \pi \chi_{\mathrm{c}}(\omega)\right] k a k b I_{m}^{\prime}(k b) K_{m}(k a) I_{m}(k b) K_{m}^{\prime}(k a), \\
\alpha= & 1+4 \pi \chi_{\mathrm{c}}(\omega) k b I_{m}^{\prime}(k b) K_{m}(k a)-4 \pi \chi_{\mathrm{s}}(\omega) k a I_{m}(k a) K_{m}^{\prime}(k a) \\
& +(4 \pi)^{2} \chi_{\mathrm{s}}(\omega) \chi_{\mathrm{c}}(\omega) k a k b\left[I_{m}^{\prime}(k b) K_{m}(k a) I_{m}(k b) K_{m}^{\prime}(k a)-I_{m}(k a) K_{m}^{\prime}(k a) I_{m}^{\prime}(k b) K_{m}(k b)\right] .
\end{aligned}
$$

The determinant $|B|=0$ gives the quadratic equation for $\omega(\omega+i \gamma)$ for localized surface plasmon frequencies that are given by

$$
\omega_{ \pm}\left(\omega_{ \pm}+i \gamma\right)=\omega_{\mathrm{p}}^{2} \frac{-\beta \pm \sqrt{\beta^{2}-4 \alpha \eta}}{2 \alpha}
$$

When the nanoshell with dielectric core exists in vacuum or air, i.e., $\chi_{\mathrm{s}}(\omega)=0$, the surface plasmon frequencies are given ignoring the plasmon decay $\gamma=0$ at $m=1$ and $k=0$ by Eq. (22).

\section{References}

[1] S. A. Maier, Plasmonics: Fundamentals and Applications (Springer, New York, 2007).

[2] M. A. Garcia, J. Phys. D 44, 283001 (2011).

[3] M. I. Stockman, Opt. Express 19, 22029 (2011).

[4] N. J. Halas, S. Lal, W.-S. Chang, S. Link, and P. Nordlander, Chem. Rev. 111, 3913 (2011).

[5] S. Hayashi and T. Okamoto, J. Phys. D 45, 433001 (2012).
[6] G. Raschke, S. Brogl, A. S. Susha, A. L. Rogach, T. A. Klar, J. Feldmann, B. Fieres, N. Petkov, T. Bein, A. Nichtl, and K. Kürzinger, Nano Lett. 4, 1853 (2004).

[7] C. L. Nehl, N. K. Grady, G. P. Goodrich, F. Tam, N. J. Halas, and J. H. Hafner, Nano Lett. 4, 2355 (2004).

[8] F. Tam, C. Moran, and N. Halas, J. Phys. Chem. B 108, 17290 (2004).

[9] E. Prodan, C. Radloff, N. J. Halas, and P. Nordlander, Science 302, 419 (2003).

[10] E. Prodan and P. Nordlander, Nano Lett. 3, 543 (2003). 
[11] F. J. García de Abajo, J. Phys. Chem. C 112, 17983 (2008).

[12] K. Tanabe, J. Phys. Chem. C 112, 15721 (2008).

[13] N. K. Pathak, Parthasarathi, P. S. Kumar, and R. P. Sharma, Phys. Chem. Chem. Phys. 21, 9441 (2019).

[14] S. Raza, G. Toscano, A.-P. Jauho, N. A. Mortensen, and M. Wubs, Plasmonics 8, 193 (2012).

[15] S. Raza, S. I. Bozhevolnyi, M. Wubs, and N. A. Mortensen, J. Phys.: Condens. Matter 27, 183204 (2015).

[16] B. Vasvári, Phys. Rev. B 55, 7993 (1997).

[17] K. Sasaki, S. Murakami, and H. Yamamoto, Appl. Phys. Lett. 108, 163109 (2016).

[18] M. S. Ukhtary and R. Saito, Carbon 167, 455 (2020).

[19] A. V. Kabashin, P. Evans, S. Pastkovsky, W. Hendren, G. A. Wurtz, R. Atkinson, R. Pollard, V. A. Podolskiy and A. V. Zayats, Nat. Mater. 8, 867 (2009).

[20] M. Ichikawa, J. Phys. Soc. Japan 80, 044606 (2011).

[21] M. Ichikawa, e-J. Surf. Sci. Nanotechnol. 12, 431 (2014).

[22] M. Ichikawa, e-J. Surf. Sci. Nanotechnol. 13, 391 (2015).

[23] M. Ichikawa, Condens. Matter 1, 9 (2016).

[24] M. Ichikawa, e-J. Surf. Sci. Nanotechnol. 15, 103 (2017).

[25] M. Ichikawa, e-J. Surf. Sci. Nanotechnol. 16, 329 (2018).

[26] M. Ichikawa, Jpn. J. Appl. Phys. 58, SIIA07 (2019).

[27] M. Ichikawa, e-J. Surf. Sci. Nanotechnol. 18, 190 (2020).

[28] K. Morawetz, P. Lipavský, and M. Schreiber, Phys. Rev. B 72, 233203 (2005).
[29] Y. Pavlyukh, J. Berakdar, and K. Köksal, Phys. Rev. B 85, 195418 (2012).

[30] N. A. Mortensen, P. A. D. Goncalves, F. A. Shuklin, J. D. Cox, C. Tserkezis, M. Ichikawa, and C. Wolff, Nanophotonics (2021), DOI: 10.1515/nanoph-2021-0084.

[31] E. Prodan and P. Nordlander, Chem. Phys. Lett. 352, 140 (2002).

[32] C. Oubre and P. Nordlander, J. Phys. Chem. B 108, 17740 (2004).

[33] E. Prodan and P. Nordlander, Chem. Phys. Lett. 349, 153 (2001).

[34] M. Brack, Rev. Mod. Phys. 65, 677 (1993).

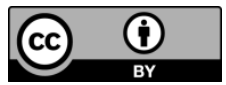

All articles published on e-J. Surf. Sci. Nanotechnol. are licensed under the Creative Commons Attribution 4.0 International (CC BY 4.0). You are free to copy and redistribute articles in any medium or format and also free to remix, transform, and build upon articles for any purpose (including a commercial use) as long as you give appropriate credit to the original source and provide a link to the Creative Commons (CC) license. If you modify the material, you must indicate changes in a proper way.

Published by The Japan Society of Vacuum and Surface Science 